\title{
Manifestations of climate change from the point of view of farmers in one of the villages of Desouk district, Kafr El-Sheikh governorate, Egypt
}

\author{
Elbarky Sama F.* \\ Agricultural Extension and Rural Development Research Institute, Agricultural Research Center, Giza, Egypt
}

\begin{abstract}
This research was conducted in the village of Kafr Majar, Desouk district, with the aim of clarifying the manifestations of climate change from the farmer's perspective in the research area, identifying the relative importance of these dimensions, and finally to identify the relationship between those aspects and some independent variables. The village of Kafr Majar was chosen randomly from the villages of Desouk district, and the owners of the village were (300) farmers. A total of 168 farmers were sampled. The data were collected using a questionnaire form through a personal interview with the sample members after conducting the initial test on it, during January 2020, and iterations, arithmetic mean, weighted average, correlation and regression analyze, were used to analyze and present the data. The most important results that were reached were identifying the relative importance of the three dimensions of the manifestations of climate change arranged according to the weighted average as follows: The dimension of the manifestations of climate change related to water is in the first, followed by the dimension of the aspects related to the environment in the second, and finally the dimension related to the aspects related to agricultural land and crop quality. The nine independent variables studied together explain $(47.9 \%)$ of the variation in the manifestations of climate change from the perspective of the farmers, and that the most contributing variables in explaining the variation in the manifestations of climate change are achievement motivation, followed by the attitude toward change, then the educational status of the respondent.
\end{abstract}

Keywords: manifestations, climate change, farmers, Kafr El-Sheikh.

\footnotetext{
* Corresponding author: Elbarky Sama F.,
}

E-mail address: re_rakha@yahoo.com 
مظاهر التغير ات المناخية من وجهة نظر الزراع بإحدى قرى مركز دسوق محافظة كفر الثيخ بجمهورية مصر العربية

\author{
سماء فاروق البرقي \\ معهد بحوث الارشاد الزر اعى و التنمية الريفية، مركز البحوث الزراعية، الجيزة، جمهورية مصر العربية
}

أجري هذا البحث بقرية كفر مجر بمركز دسوق بهدف التعرف على مظاهر التغيرات المناخية من وجهة نظر الزراع كهذف رئيسي للبحث،

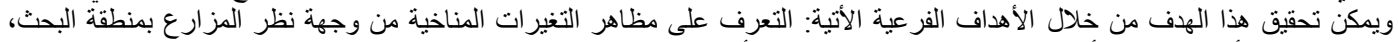

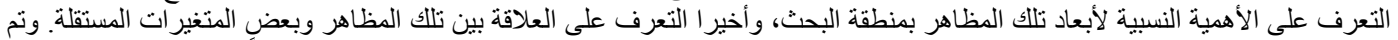

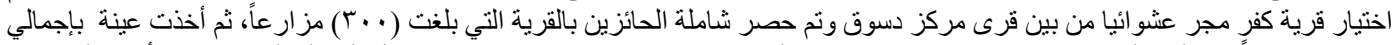

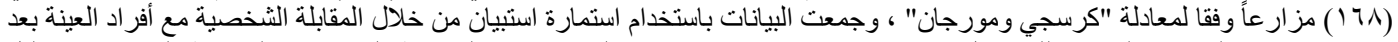

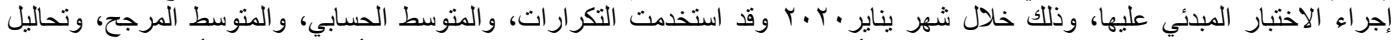

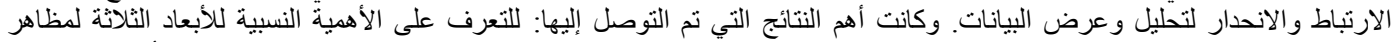

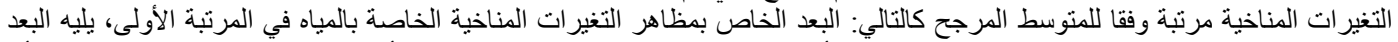

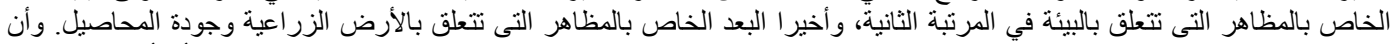

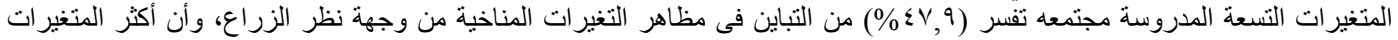
إسهاما فى تفسير التباين فى مظاهر التغيرات المناخية هى دافعية الإنجاز, يليه الاتجاه نحو التغيير ، ثم الحالة التعليمية للمبحوث. 


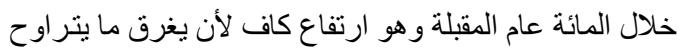

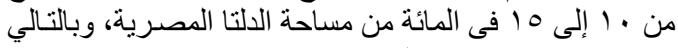

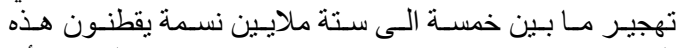

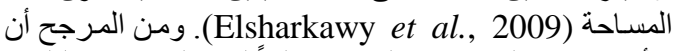

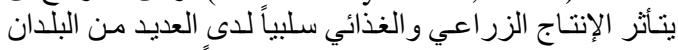

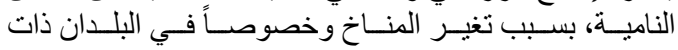

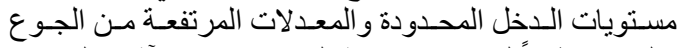

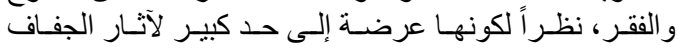

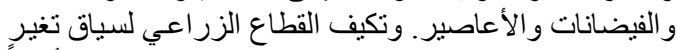

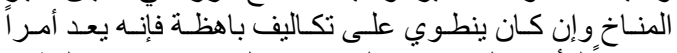

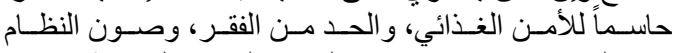

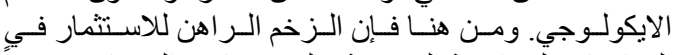

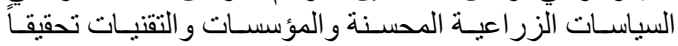

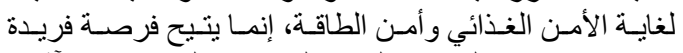

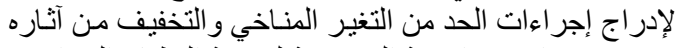

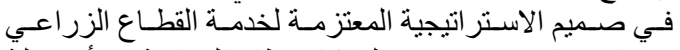

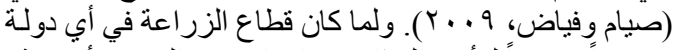

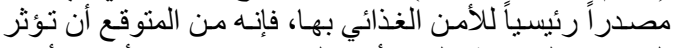

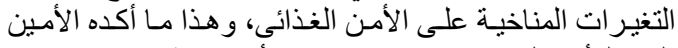

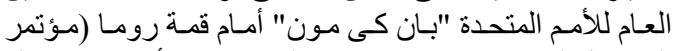

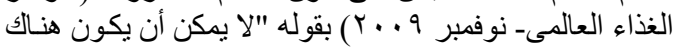

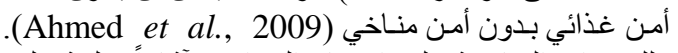

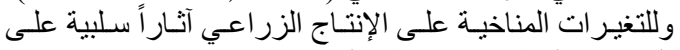

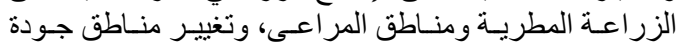

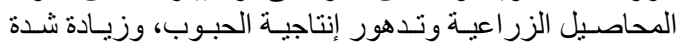

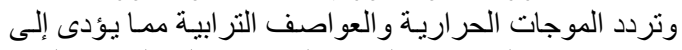

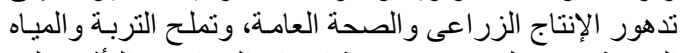

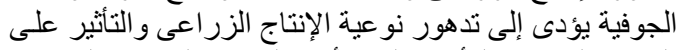

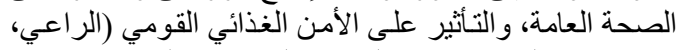

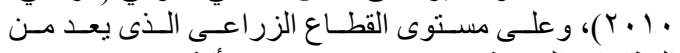

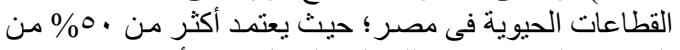

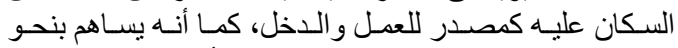

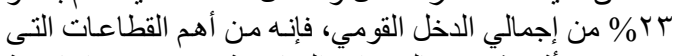

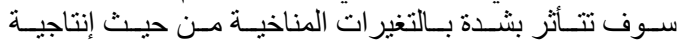

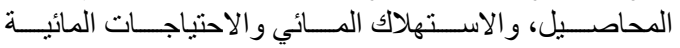

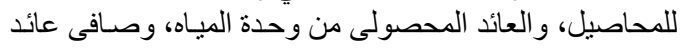

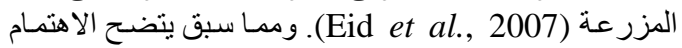

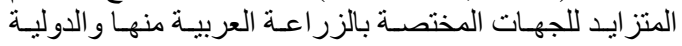

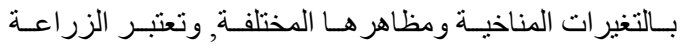

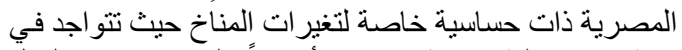

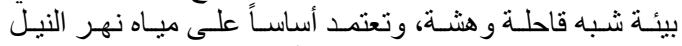

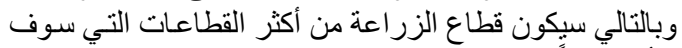

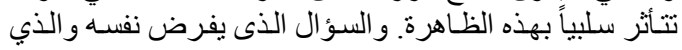

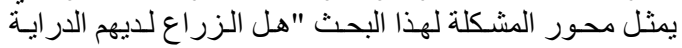

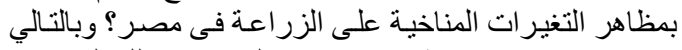

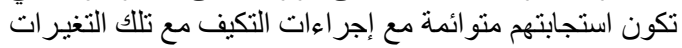

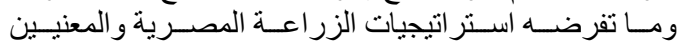
بالتغير ات المناخية بالمر اكز البحثية، حيث تمثل معرفتهم بتلكي
مقدمة - - مقة

يهتم العالم اليوم بظاهرة التغير ات المناخية كظاهرة عالميـة لهـا

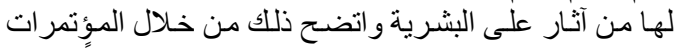

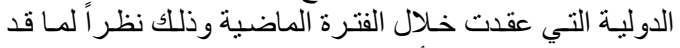

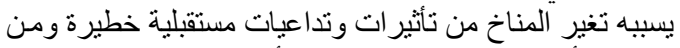

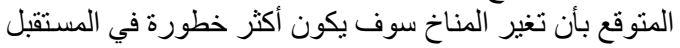

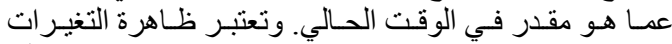

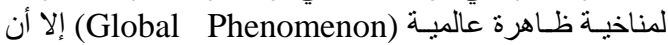

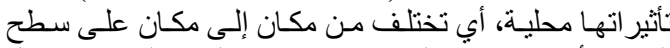

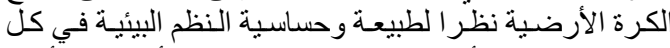

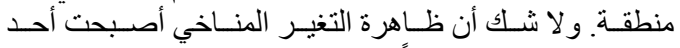

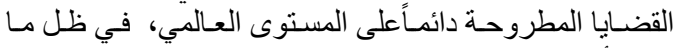

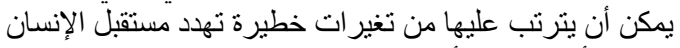

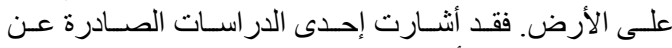

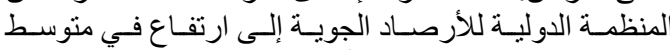

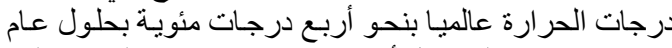

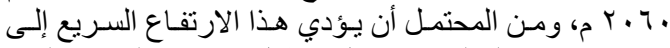

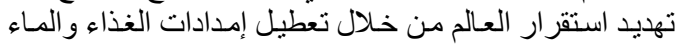

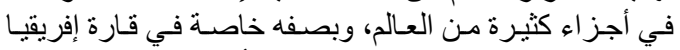

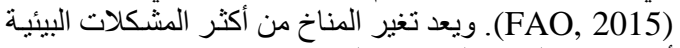

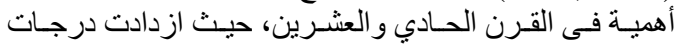

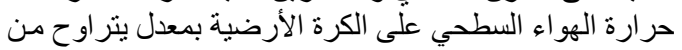

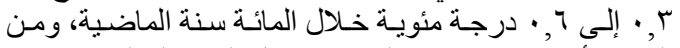

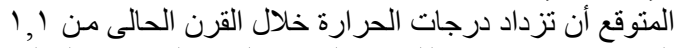

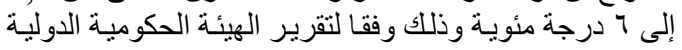

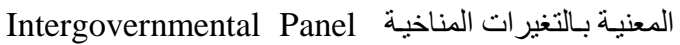
on Climate Change (IPCC, 2007)

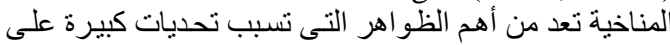

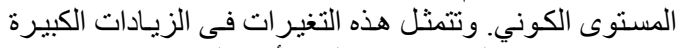

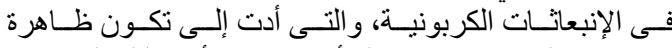

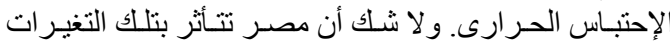

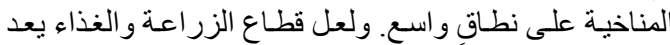

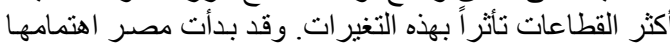

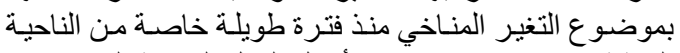

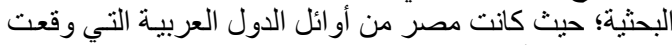

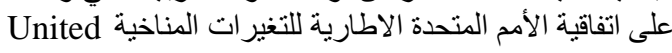
Nation Framework Convention on Climate في Change (UNFCCC)

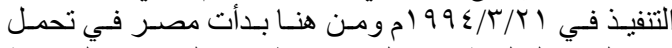

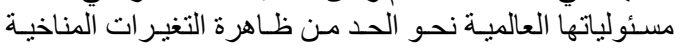

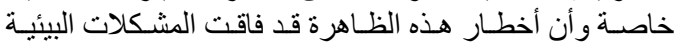

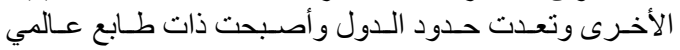

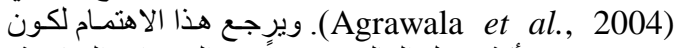

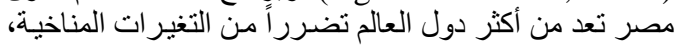

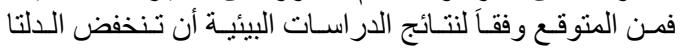

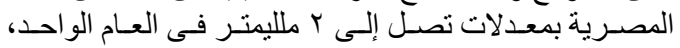

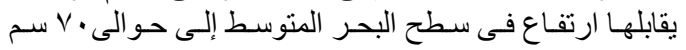




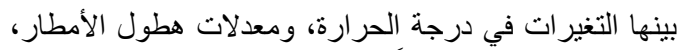

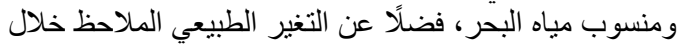

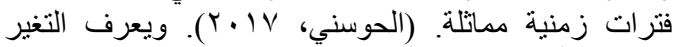

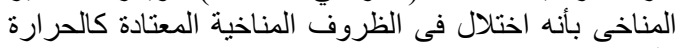

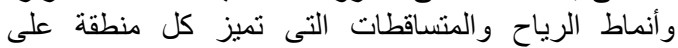

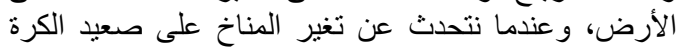

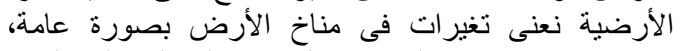

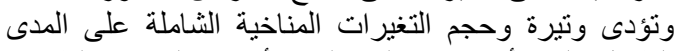

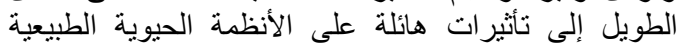

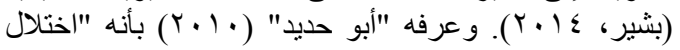

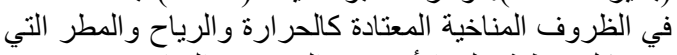

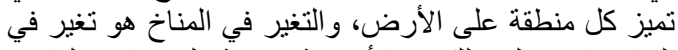

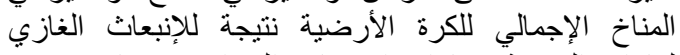

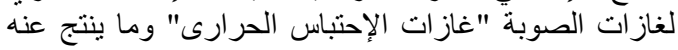

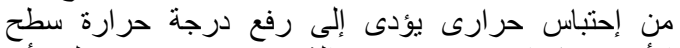

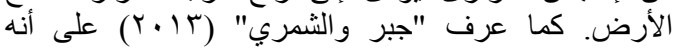

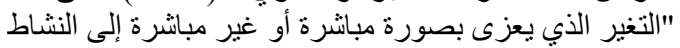

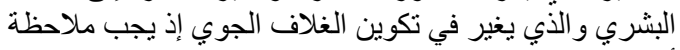

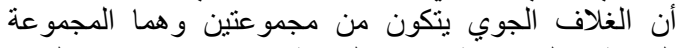

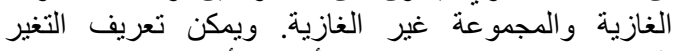

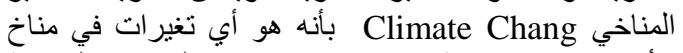

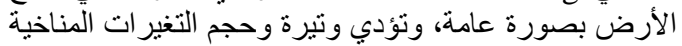

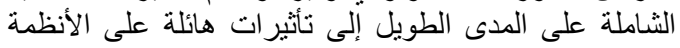

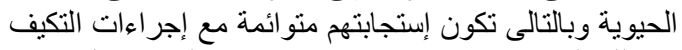

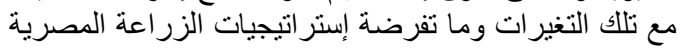
والمعنيين بالتغير ات المناخية بالمر اكز البحثية بيعية، البزاتية (داوود،

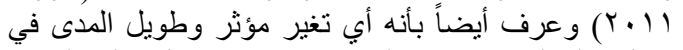

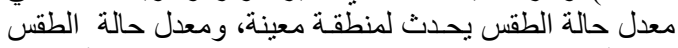

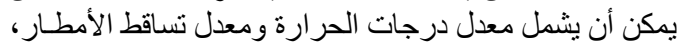

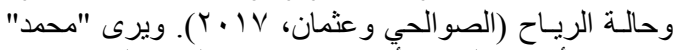

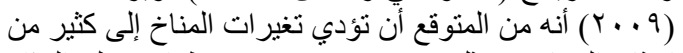

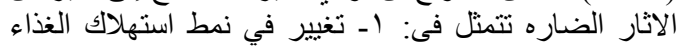

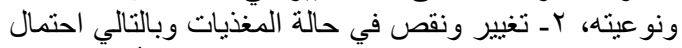
حدوث تغيرات في صحة البشر خاصة النساء و والأطفال وكبار النيار

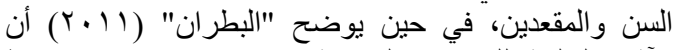
الآثار السلبية للتغيرات المناخية تنحصر في: تغير التير منوسط

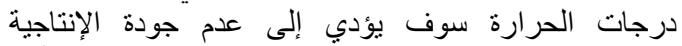

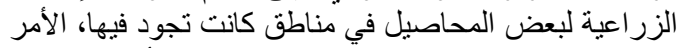

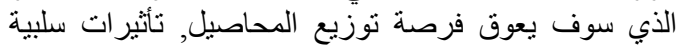

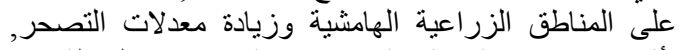

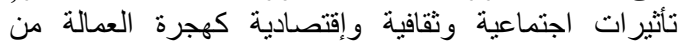

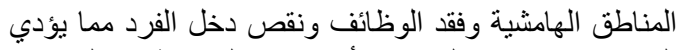

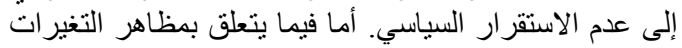

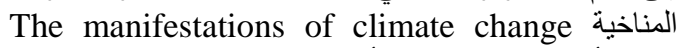

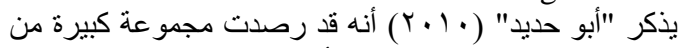

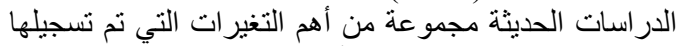

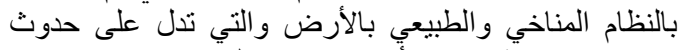

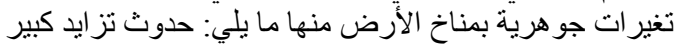

المظاهر محوراً أساسياً للتكيف مع تلك المظاهر.

\section{أهداف البحث}

o التعرف على مظاهر التغير ات المناخية من وجهة نظر الزراع بمنطقه البحث. التهي.

o التعرف على الأهمية النسبية لأبعاد تلك المظاهر بمنطقة البحث.

o التعرف على العلاقة بين أبعاد مظاهر التغير ات المناخية وبعض المتغير ات المستقلة.

\section{الإطار النظري}

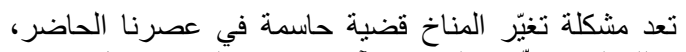

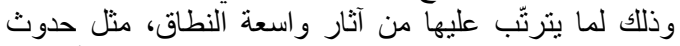

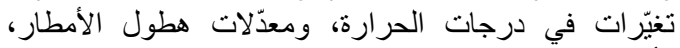

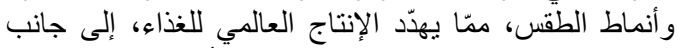

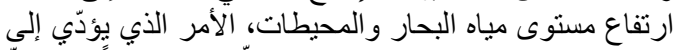

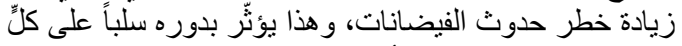

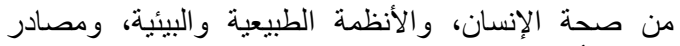

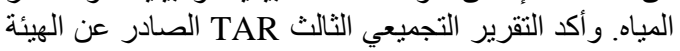

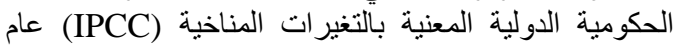

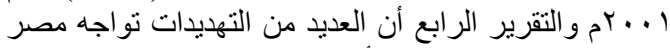

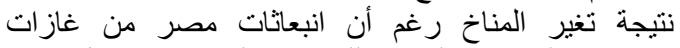

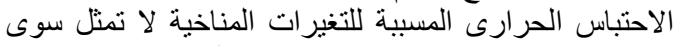

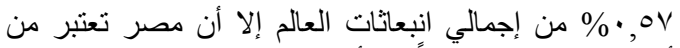

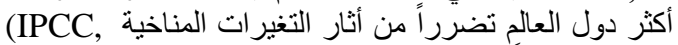

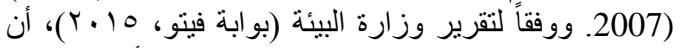

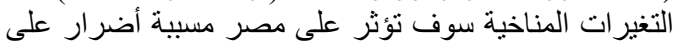

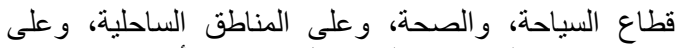

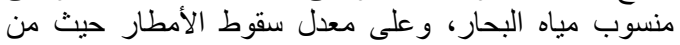

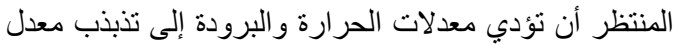

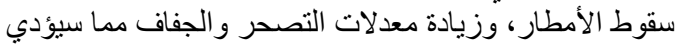

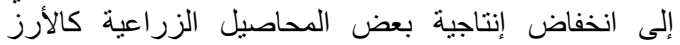

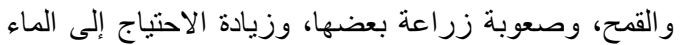

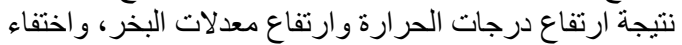

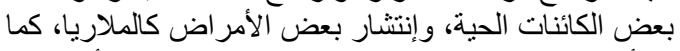

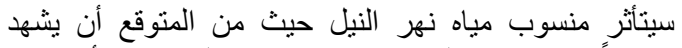

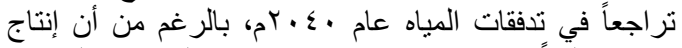

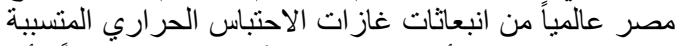

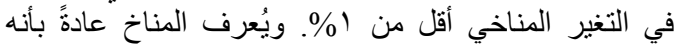

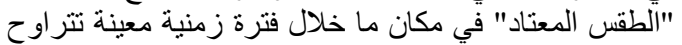

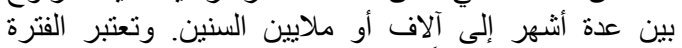

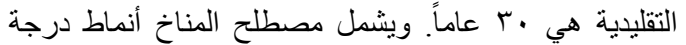

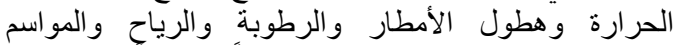

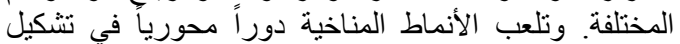

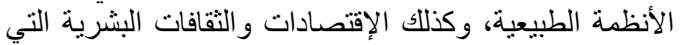
تعتمد عليها. يتمثل التغير المناخي في عدة طرق مختلفة، من الإنية 
الأحفوري من المواد الأحفورية كالفحم الحجري، و الفحم

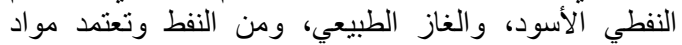

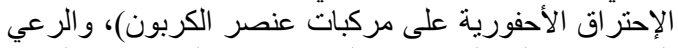

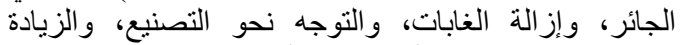

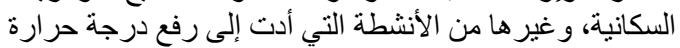

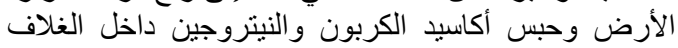

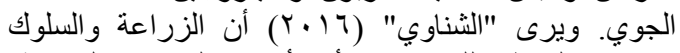

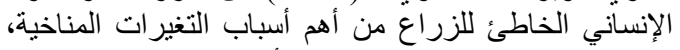

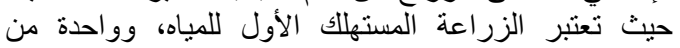

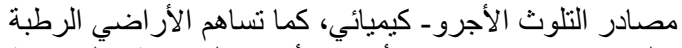

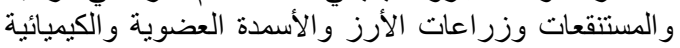

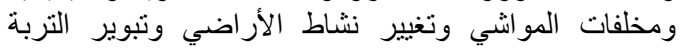

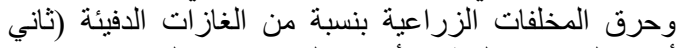
أكسيد الكربون و الميثان و أكاسيد النيتروجين بنين والكبر الغيريت).

\section{الفروض البحثية}

تحقيقاً للهدف الثالث للبحث أمكن صـياغة الفروض البحثيـة التالبة : تحفة

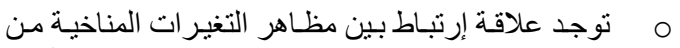

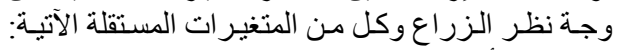

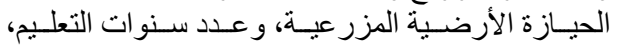

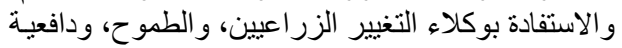

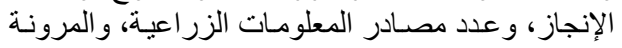

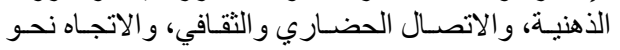
التغيير. تسهم المتغير ات المستقلة السابقة إسهاماً معنوياً فى تفسير

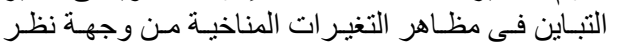

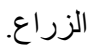

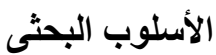

ويتضمن شاملة و عينة البحث، وأسلوب جمع وتحليل البيانات,

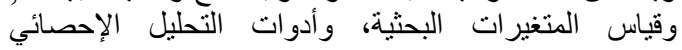
ونعرض كل منها كالتالي:

ا ـ شاملة وعينة البحث: أجرى هذا البحث بقرية كفر مجر

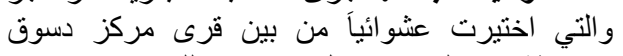

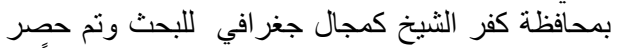

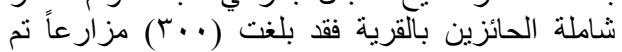

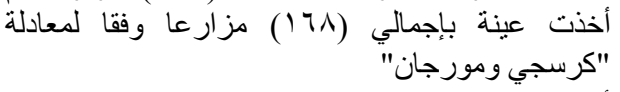

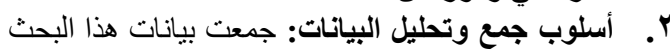

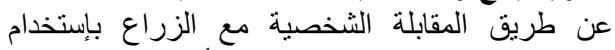

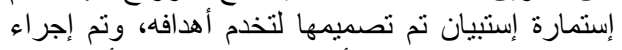

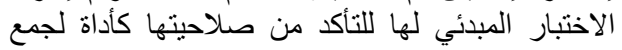

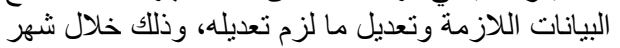

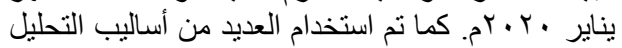

بالقيمة المتوسطة اليومية لفارق درجات الحرارة ما بين درجة

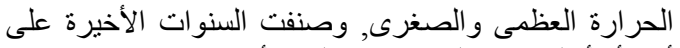

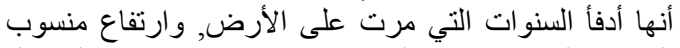

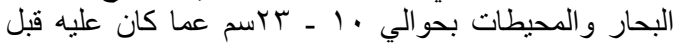

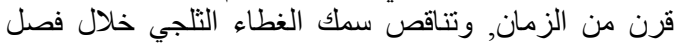

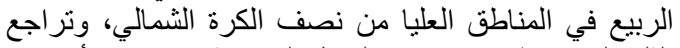

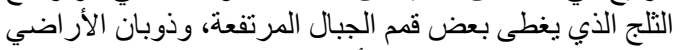

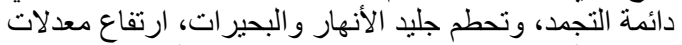

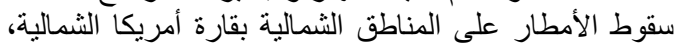

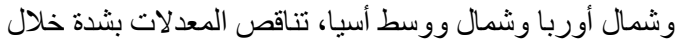

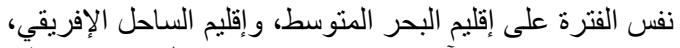

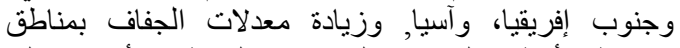

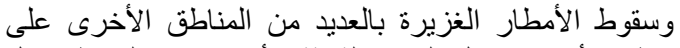

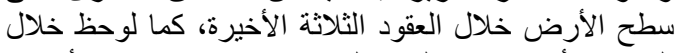

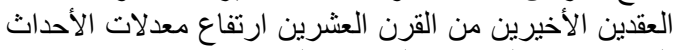

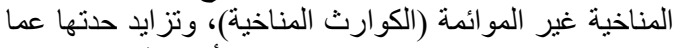

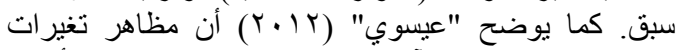

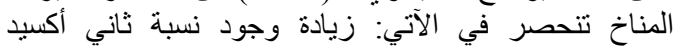

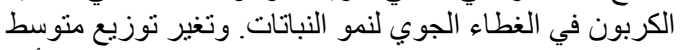

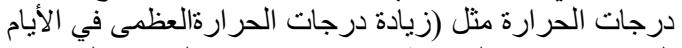

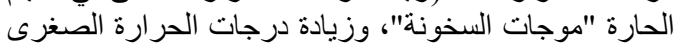

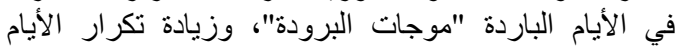

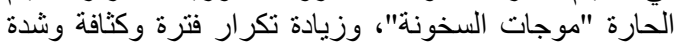

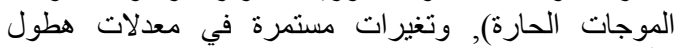

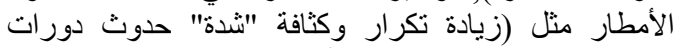

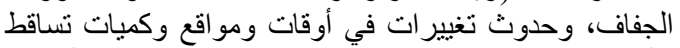

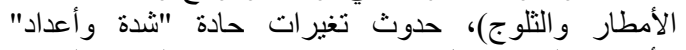

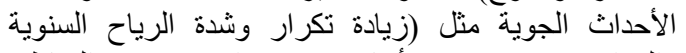

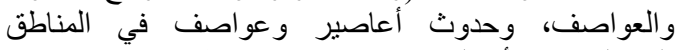

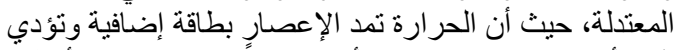

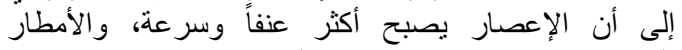

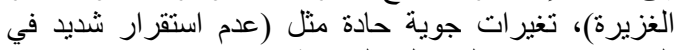

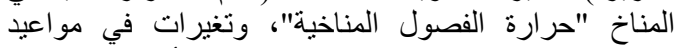

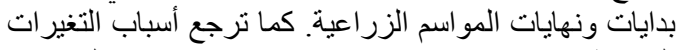

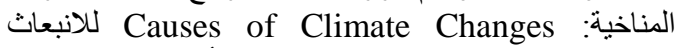
الغازي وما ينتج عنه من احتباس حراري أدى إلى الى رفع درجة الإنة

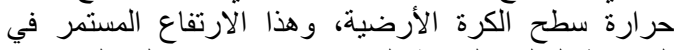

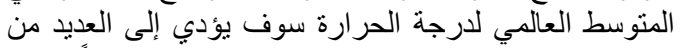

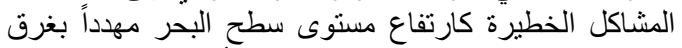

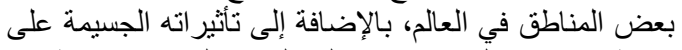

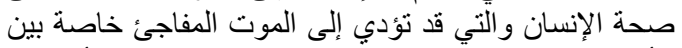

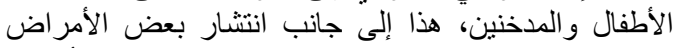

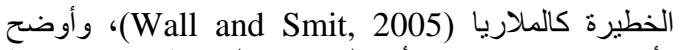

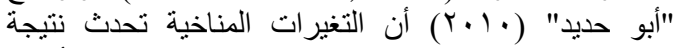

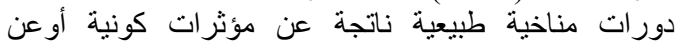

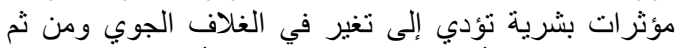

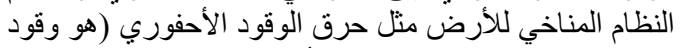

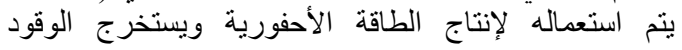




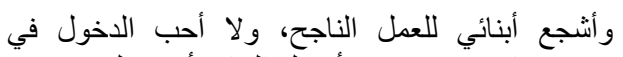

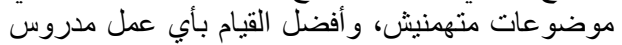

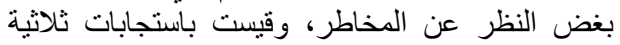

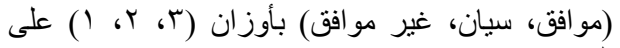
الترتيب.

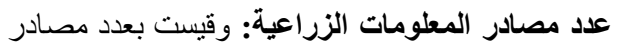

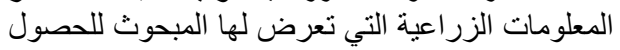

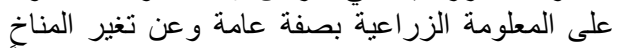

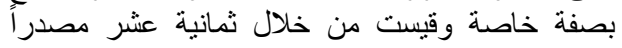

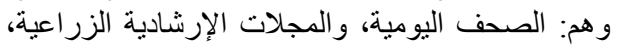

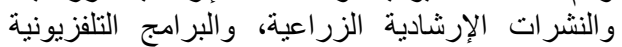

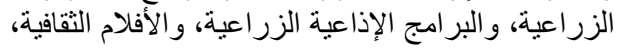

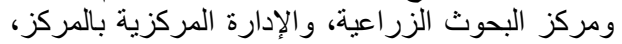

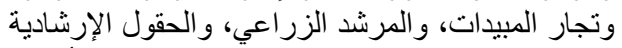

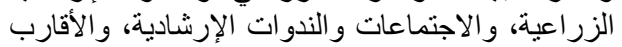

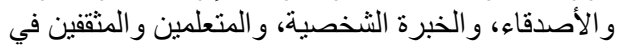

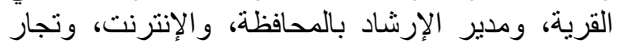

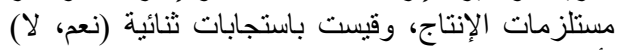

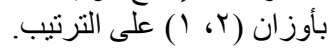

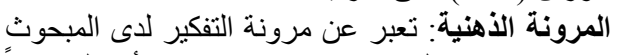

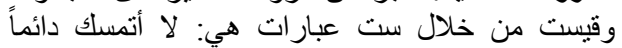

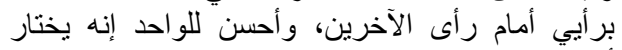

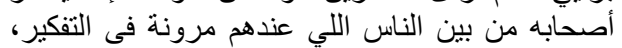

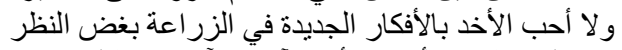

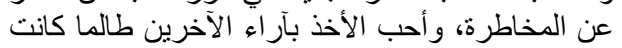

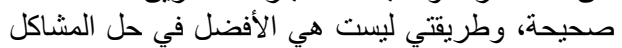

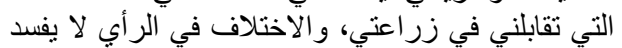

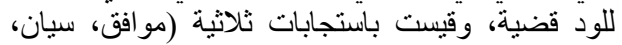

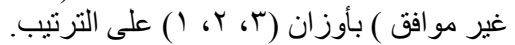

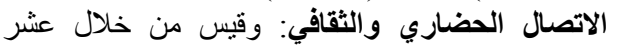

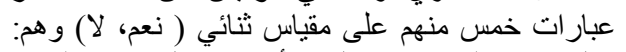

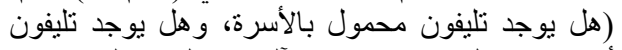

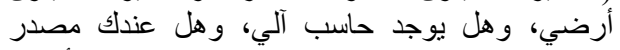

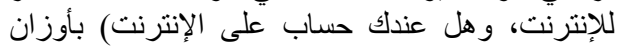

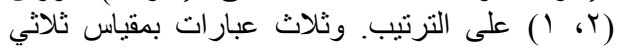
(عالية، متوسطة، منخفضة) و هم (مدى ايجاد استخدام

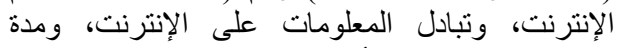

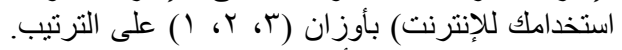

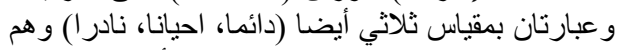

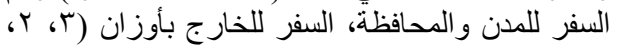
( ) على الترتيب.

الاتجاه نحو التغيير: ويعبر عن رأي المبحوث عن عن التغيير من

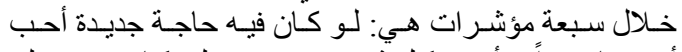

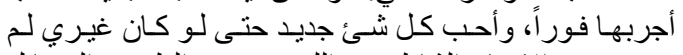

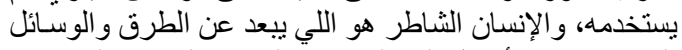

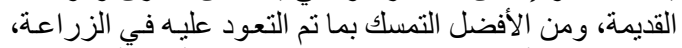

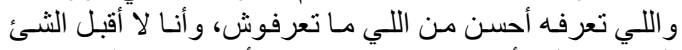

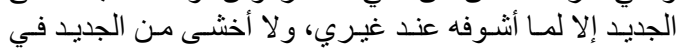

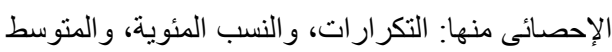

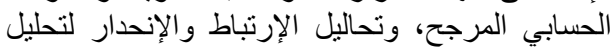
وعرض البيانات.

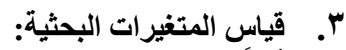

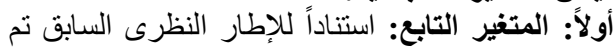

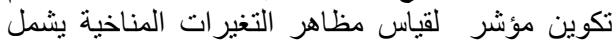

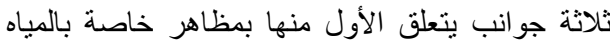

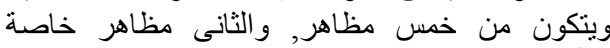
بالأرض الزر اعية وجودة المحاصيل ويتكون من ثمانية

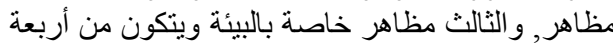

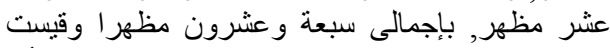

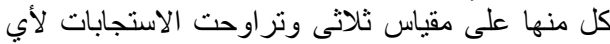

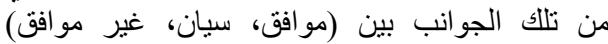

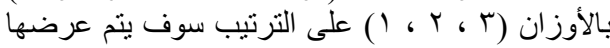

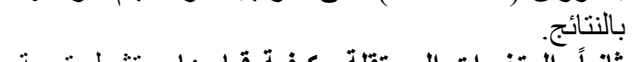
ثانياً: المتغيرات المستقلة وكيفية قياسها: وتثمل تسعة

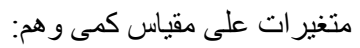

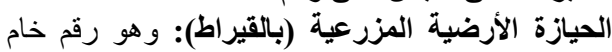

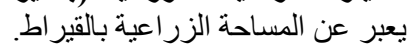

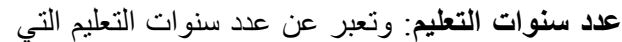

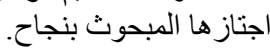

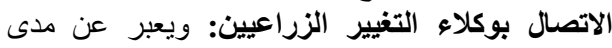

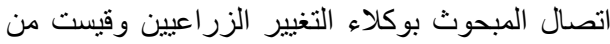

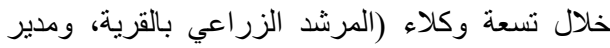

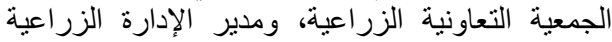

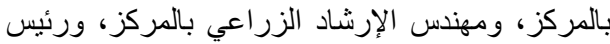

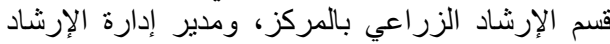

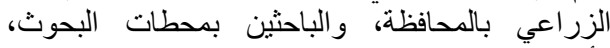

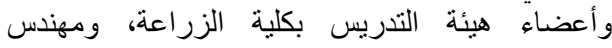
المكافحة بالمديرية)، وقيست باستجابات ثنائية (نعم، لا لاعة )

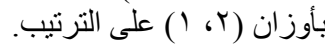
الطموح: وقيس من خلال عثر عبار ات تعبر التير عن طموح

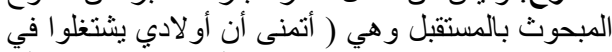

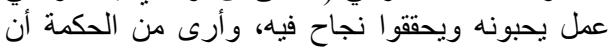

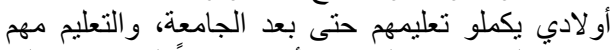

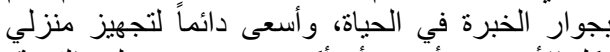

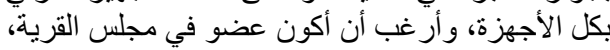

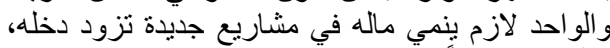

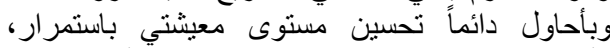

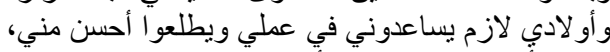

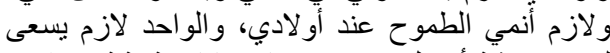

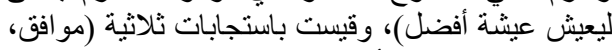

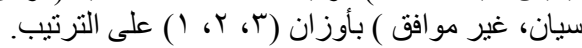

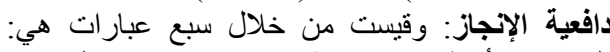

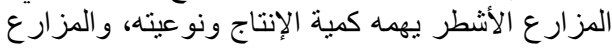

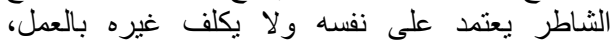

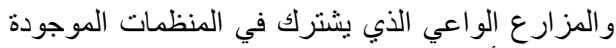

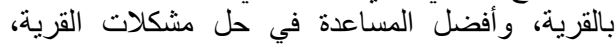




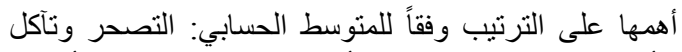

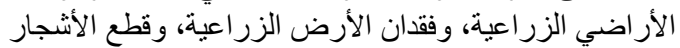

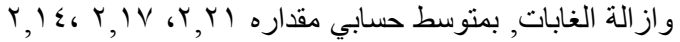

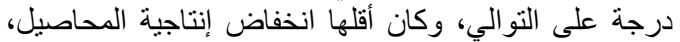

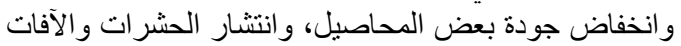

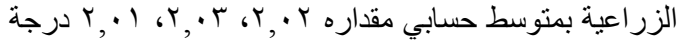

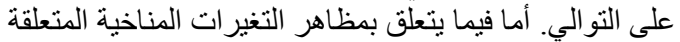

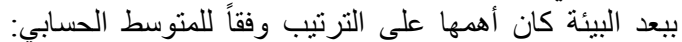

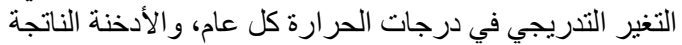

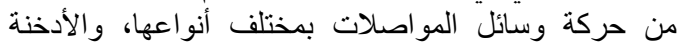

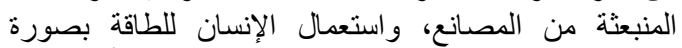

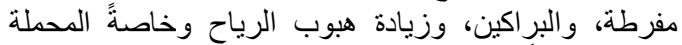

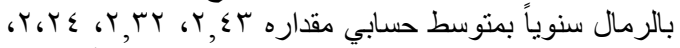

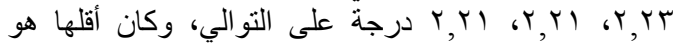

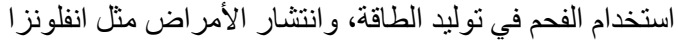

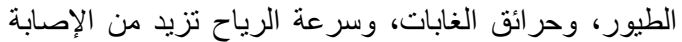

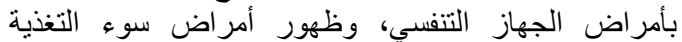

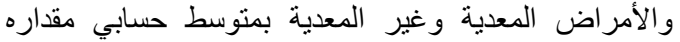

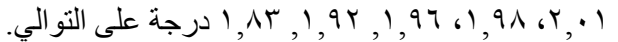

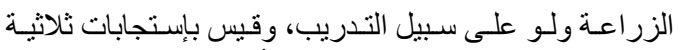

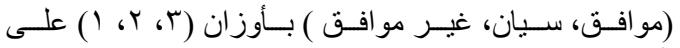

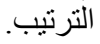

\section{النتائج ومناقشتها}

أولا: النتائج الخاصة بتوزيع مظاهر التغيرات المناخية وجهة نظر الزراع العزاع

أوضحت النتائج بالجدول (1) أن توزيع مظاهر التغيرات

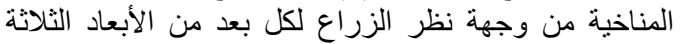

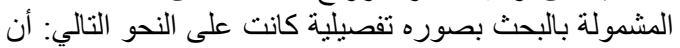

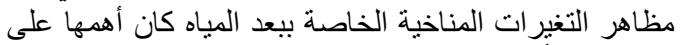

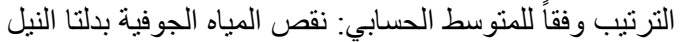

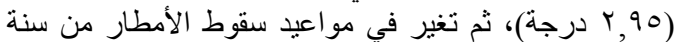

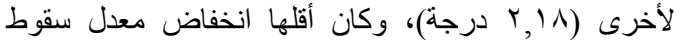

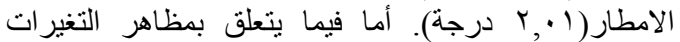

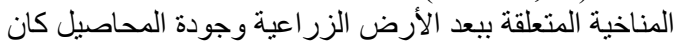

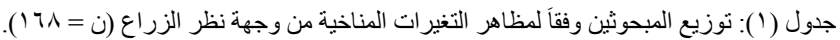

\begin{tabular}{|c|c|c|c|c|c|c|c|}
\hline \multirow{2}{*}{ المتوسط الحسابي المرجح } & \multicolumn{2}{|c|}{ 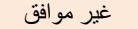 } & \multicolumn{2}{|c|}{ سيان } & \multicolumn{2}{|c|}{ مو افق } & \multirow{2}{*}{ مظاهر التغيرات المناخية } \\
\hline & $\%$ & العدد & $\%$ & العدد & $\%$ & العدد & \\
\hline \multicolumn{8}{|r|}{ أ. مظاهر التغبر ات المناخية التى تتعلق بالمياه } \\
\hline$r, 1 \Lambda$ & $T \cdot, r$ & $\Gamma \Sigma$ & $\Sigma 1,1$ & 79 & $r \Lambda, \gamma$ & 70 & 1. تغير في مو اعيد سقوط الامطار من سنة لاخرى \\
\hline$r, 11$ & $T \cdot, r$ & $\Gamma \varepsilon$ & $\varepsilon \wedge, \wedge$ & Ar & $\Gamma, \cdot$ & or & r. تُغير في كميات الامطار من سنة لأخرى \\
\hline T,IT & 11,0 & $\Pi$ & $0 ., 7$ & 10 & $\mu, \cdot$ & or & بـ انخفاض مستوى الماء في نهر النيل \\
\hline$r, 90$ & $T_{T}, Y^{\prime}$ & rq & $\varepsilon Y, r$ & $\mathrm{y}$ & $r \varepsilon, 7$ & 01 & ع ـ نقص المياه الجوفية بدلتا النيل \\
\hline$r, \cdot 1$ & $T \cdot, \Lambda$ & ro & or, & $9 V$ & TI, & ry & 0. انخفاض معدل سقوط الامطار \\
\hline \multicolumn{8}{|c|}{ ب. مظاهر التغيرات المناخية التى تتعلق ببعد الأرض الزر اعبة وجودة المحاصيل } \\
\hline$Y, I \leqslant$ & re, & TV & $\xi 1, \mathrm{~V}$ & v. & rq, r & 71 & 1. قططع الاشجار و إز الة الغابات \\
\hline$r, r)$ & 10,0 & YY & $\varepsilon \vee, T$ & A. & $r 4,9$ & TY & Y. التصحر وتأكل الأر اضي الزراعبة \\
\hline$\overline{T, I V}$ & YY,T & $\Gamma \Lambda$ & $\varepsilon \vee$, & $\mathrm{V9}$ & $r \cdot, \xi$ & 01 & بـ آ فقدان الأرض الزر اعية \\
\hline$r, \cdot \varepsilon$ & $r 7,1$ & $\leqslant 0$ & $\varepsilon r, q$ & VY & $r \cdot, \Sigma$ & 01 & ع. انخفاض خصوبة الأرض الزر اعية \\
\hline$r, \cdot \Lambda$ & $T V, \varepsilon$ & $\sum \uparrow$ & $\varepsilon 0,1$ & VV & $r \bar{r}, \Lambda$ & $\varepsilon 0$ & 0. ارتفاع ملوحة الأرض \\
\hline$r_{,-T}$ & $1, T, 0$ & TI & $V T, Y$ & TK & $1 \varepsilon, r$ & TE & 7. إنخفاض إنتاجية المحاصيل \\
\hline$r_{,} \cdot r$ & IV, & rq & $T, 0$ & 1.0 & $r \cdot, r$ & $r \varepsilon$ & 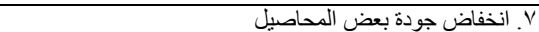 \\
\hline$r, \cdot 1$ & rY, 7 & rA & or, 7 & 9. & 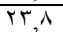 & $\varepsilon$. & يـ انتشار الحشر ات والآفات الزراعية \\
\hline \multicolumn{8}{|r|}{ جـــ مظاهر التغبرات المناخبة التى تتعلق ببعد البيئة } \\
\hline$r, Y)$ & $1 \pi, 1$ & rr & or,,$\varepsilon$ & $\mathrm{A \Lambda}$ & $r \varepsilon, 0$ & 01 & ا. البر اكين \\
\hline 1,97 & $r T, \Lambda$ & $\varepsilon \cdot$ & 07, & $9 \leq$ & $r \cdot, r$ & $r \varepsilon$ & 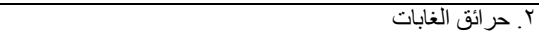 \\
\hline$r, \cdot \Lambda$ & 19,7 & ru & or,,$\varepsilon$ & $\mathrm{A \Lambda}$ & rA, $\cdot$ & $\sum V$ & 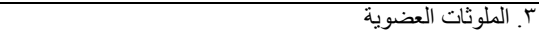 \\
\hline$r, Y \varepsilon$ & $1 \pi, y$ & Tr & $\varepsilon \wedge, \wedge$ & Ar & rV, & $7 \pi$ & ع. الأدخنة المنبعثة من المصانع \\
\hline t, rt & $19, \cdot$ & re & $r \cdot, \varepsilon$ & 01 & $0 ., 7$ & 10 & •. الأدخنة الناتجة من حركة وسائل المو اصلات بمختلف أنو اعها \\
\hline T, Y T & $1+, \mathrm{V}$ & rT & $\leqslant १, \varepsilon$ & Ar & rq,9 & TY & 7. استعمال الانسان للطاقة بصورة مفرطة \\
\hline$r, 1$. & 11,0 & TI & or, & 19 & $r \wedge, 7$ & $\varepsilon \wedge$ & 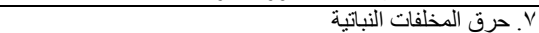 \\
\hline$r, \cdot 1$ & $I V, r$ & rq & $7 \leqslant, 9$ & 1.9 & $1 v, 9$ & $r \cdot$ & ^ـ. استخدام الفحم في توليد الطاقة \\
\hline$r, \cdot 0$ & TI, & T4 & 01,1 & AV & $r\rceil, \wedge$ & $\varepsilon 0$ & 9. الغاز ات المنبعثة من اجهزة التكييف و المبيدات الحشرية \\
\hline$r, \leqslant$ & $\mathrm{v}, \mathrm{v}$ & $\pi$ & «1,1 & 79 & $01, r$ & N4 & ل ا ـ التغير التدريجي في درجات الحرارة كل عام \\
\hline$r, Y$, & $\mathrm{v}, \mathrm{v}$ & 14 & $\pi$ & 1.7 & rq, r & $\leqslant 9$ & II 1. زيادة هبوب الرياح وخاصة المحملة بالرمال سنوياً \\
\hline 1,94 & YY,T & rA & $\pi, 0$ & 1.0 & $1 \varepsilon, 9$ & ro & r I. سرعة الرياح تزيد من الاصابة بأمر اض الجهاز التنفسي \\
\hline 1,91 & $r \wedge$, & $\xi V$ & $00, \varepsilon$ & 94 & 17,8 & $r \wedge$ & با I. انتشار الامر اض مثل الانفلونزا الطيور \\
\hline I, & $r \wedge, V$ & 70 & $r q, r$ & 74 & Kr, & rv & المعدية ظهور امر اض سوء التغذية و الامراض المعدية وغير \\
\hline
\end{tabular}




\section{الأخيرة.}

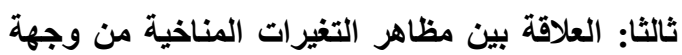

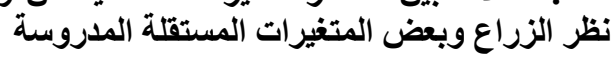

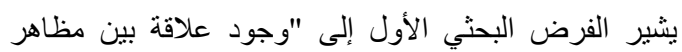

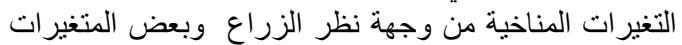

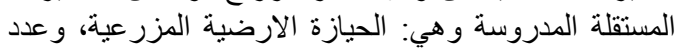

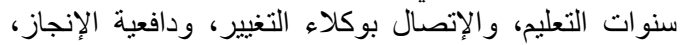

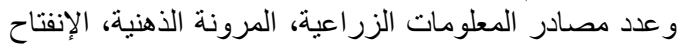
الحضاري و الثقافي، والإتجاه نحو التغيير.

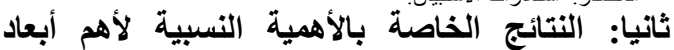

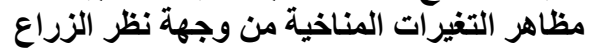

وفيما يتعلق بالأهمية النسبية لأبعاد مظاهر التغيرات المناخية

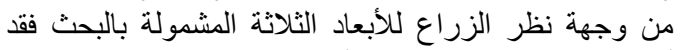

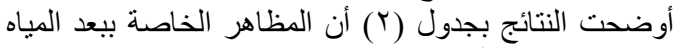

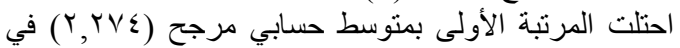

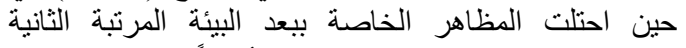

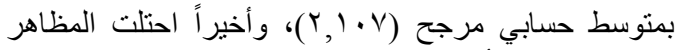
الخاصة ببعد الأرض الزراعية وجودة المحاصيل المرتبة المبل

جدول (r): الأهمية النسبية لأبعاد مظاهر التغيرات المناخية من وجهة نظر الزراع.

\begin{tabular}{|c|c|c|c|c|}
\hline التزتيب & المتوسط الحسابي المرجح & عدد البنود & المتوسط الحسابي & مظاهر التغيرات المناخية \\
\hline 1 & $T, Y V \varepsilon$ & o & 11,479 & مظاهر خاصة بالمياه \\
\hline$r$ & $1,91 \mathrm{~V}$ & $\Lambda$ & $10, Y_{11}$ & مظاهر خاصة بالأرض الزر اعبة وجودة المحاصيل \\
\hline r & $r, 1 \cdot V$ & $T \varepsilon$ & $r 9,0 .$. & مظاهر خاصة بالبيئة \\
\hline
\end{tabular}

(ץ) و الخاص بعلاقه تللك المظاهر بالمتغير ات التسعة المستقلة

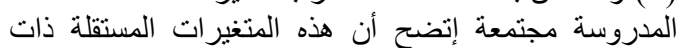

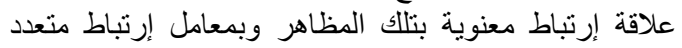

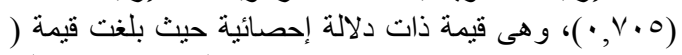

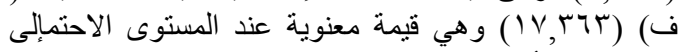

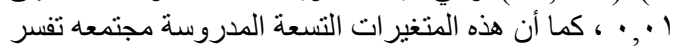

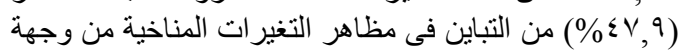

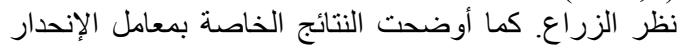

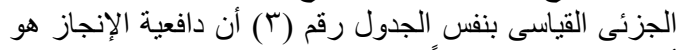

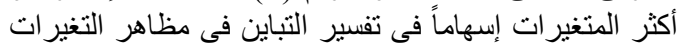

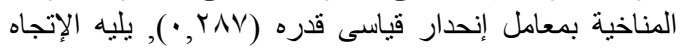

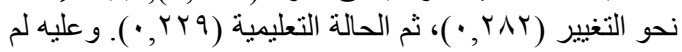
نتمكن من قبول الفرض الصفرى وقبول الفرض الفرلة البديل.

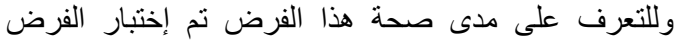

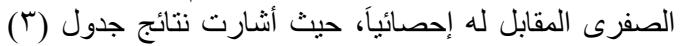

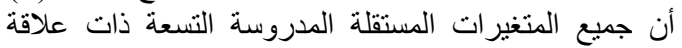

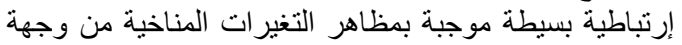

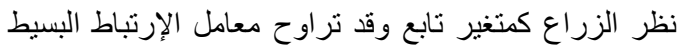

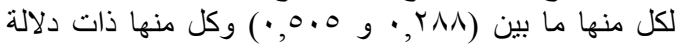

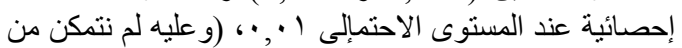

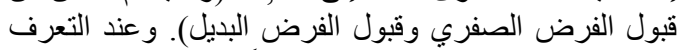

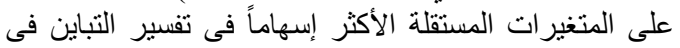

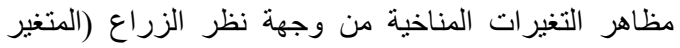

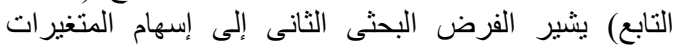

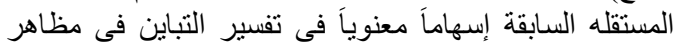

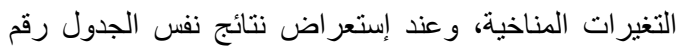

جدول (؟َ): نتائج تحليل الارتباطو الانحدار لمظاهر التغيرات المناخية من وجهة نظر الزراع والمتغيرات المستقلة المدروسة.

\begin{tabular}{|c|c|c|}
\hline معامل الانحدار الجزئي القياسي & معامل الارتباط البسيط & المتغير ات المستقلة \\
\hline$\cdot ., 09$ & 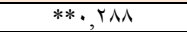 & الحيازة الأرضية المزر عية ( بالقير اط) \\
\hline **, rYq & $* * .0 .0$ & الحالة التعليمية \\
\hline$\cdot, \cdot r V$ & $* *, \leqslant V \mid$ & الاستفادة بوكلاء التغيير الزراعيين \\
\hline$\cdot, \cdot, r$ & $* *, r \leqslant \leqslant$ & الطموح ( ل الطمو \\
\hline$* * \cdot$, YAV & $* * \cdot, \xi \cdot \Lambda$ & دافعية الإنجاز \\
\hline$\cdot, 7 \wedge$ & $* *, r 94$ & عدد مصادر المعلومات الزراعية \\
\hline$\cdot, \cdot, T_{-}$ & $* *$, rvo & المرونة الذهنية \\
\hline$\cdot, \mathrm{VYT}$ & $* *,, \leqslant \leqslant 7$ & الإتصال الحضاري و الثقافي \\
\hline **., YAY & $* *,\{\leqslant 7$ & الاتجاه نحو التغيير \\
\hline \multicolumn{2}{|c|}{ معامل التحديد= } & معامل الارتباط المتعدد = V.0, V.o \\
\hline \multicolumn{3}{|c|}{ قيمة (ف) = * } \\
\hline
\end{tabular}

التغيرات المناخية من وجهة نظر الزراع أن البعد النياء

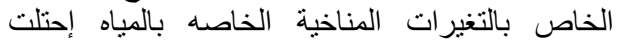
المرتبة الأولى، يليها البعد الخاص بالتيراتهيرات المناخية

\section{توصيات البحث}

1. أوضحت النتائج الخاصة بالأهمية النسبية لمظاهر 


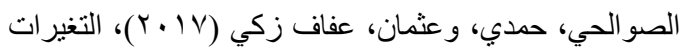

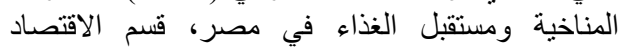

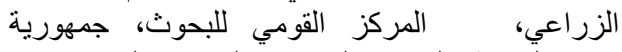

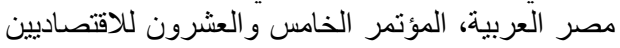

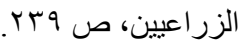

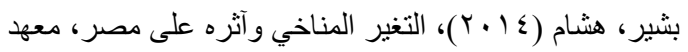

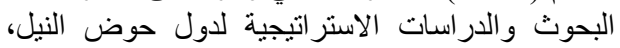

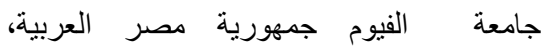
https://www.facebook.com/nilebasin.fayou .m/posts/468820449926371

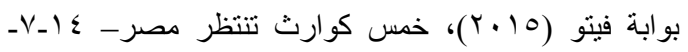

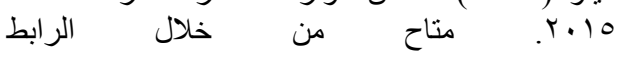
.http://www.vetogate.com/1724892

الحوسني، شيخة أحمد (Y.lV) تقرير حالة البيئة، التغير

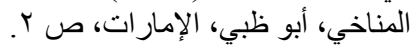

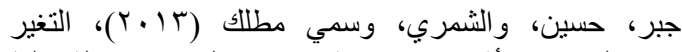

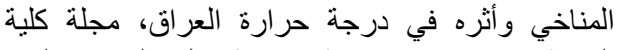

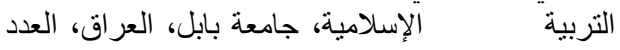

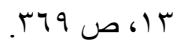

داوود، بشير عبد الحافظ (11 (Y)، التغير المناخي و الزراعة،

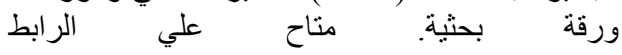
https://www.zira3a.net/articles/climate.change.html

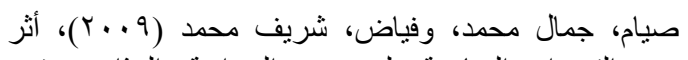

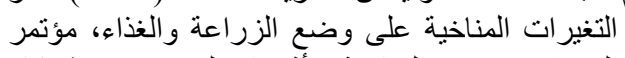

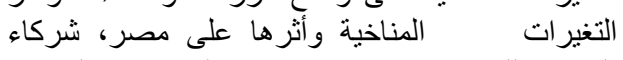

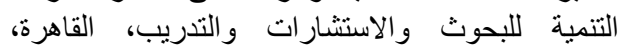

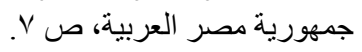

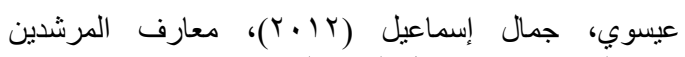

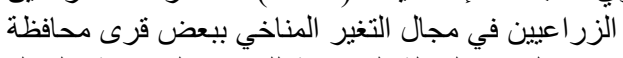

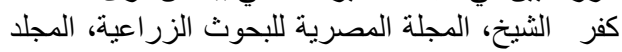

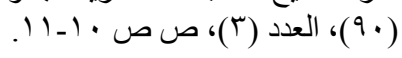

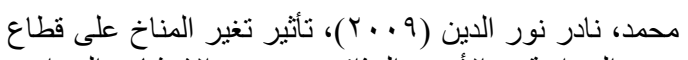

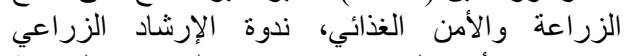

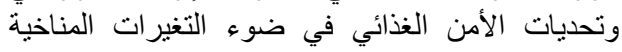

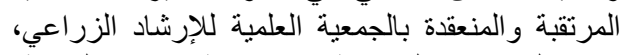

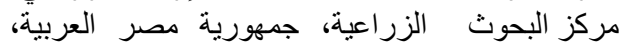

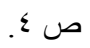

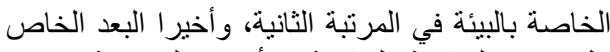

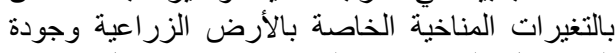

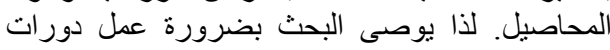

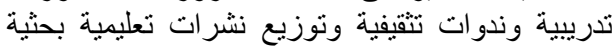

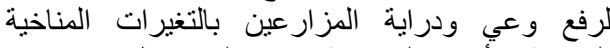
الخاصة بالأرض الزر الزية الزية وجودة المحاصيل.

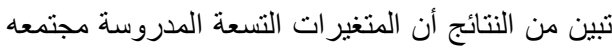

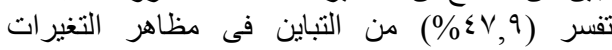

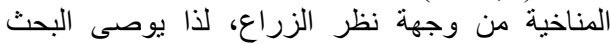

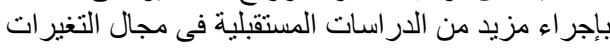

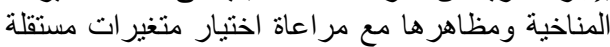

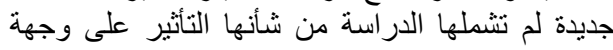

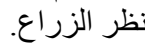
تخصيص حملات إعلامية مكئفة من قبل الدولية الدولة

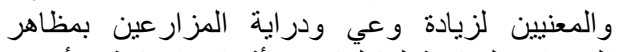

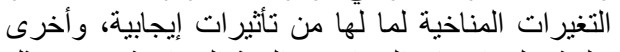

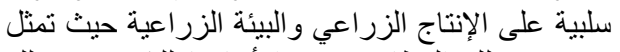

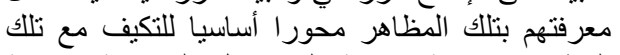
المظاهر ومن ثم حماية المحاصيل الزراعية وحماية

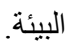

\section{قائمة المراجع}

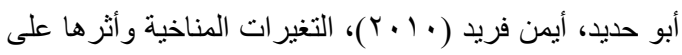

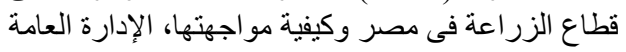

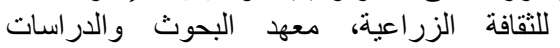
الافريقية، جامعة القاهرة، جمهورية مصر العزبة العربية، نشرة

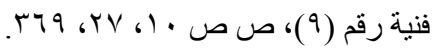

البطران، منال (1 (1) ب)، أثر تغير المناخ على الهجرة الداخلية

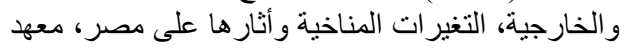

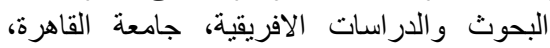

$$
\text { جمهورية مصر العربية، ص البحات الافرية، }
$$

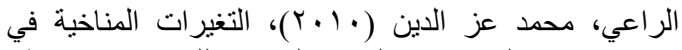

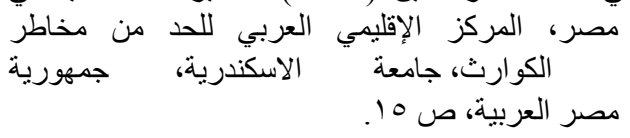

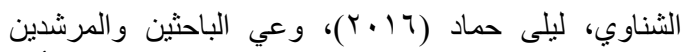

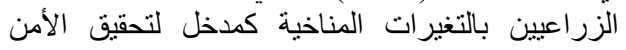
المناخي، الزراعين بالغراسة ميدانية على الباحثين بمركز

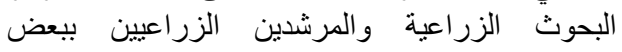

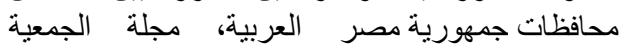
العلمية للإرشاد الزراعي، مركز البحوثة (1) البحوث الزراعية،

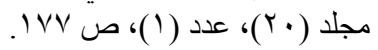




\section{المراجع الأجنبية}

Agrawala, S., Moehner, A., El Raey, M., Conway, D., Van Aalst, M., Hagenstad, M. and Smith, J. (2004), Development and climate change in Egypt: Focus on coastal resources and the Nile. Organization for Economic Co-operation and Development (OECD), Paris, France, pp.17.

Ahmed, S., Diffenbaugh, N., Hertel, T., Lobell, D., Ramankutty, N., Rios, A. and Rowhani, P. (2009), Climate volatility and poverty vulnerability in Tanzania, Policy Research Working Paper 5117, World Bank Development Research Group, World Bank, Washington, DC, USA, pp. 2.

Eid, H., El-Marsafawy, S. and Ouda, S. (2007), Assessing the economic impacts of climate change on agriculture in Egypt: A Ricardian approach. Policy Research Working Paper 4293, World Bank Development Research Group, World Bank, Washington, DC, USA, pp.3.
Elsharkawy, H., Rashed, H. and Rached, I. (2009), Climate change: the impacts of sea level rise on Egypt, $45^{\text {th }}$. ISOCARP Congress, USA, pp. 3.

FAO (2015), available online at www.fao.org/climalechange/unfccc.bonn2015 .

IPCC (2007), Climate change the physical science basis, contribution of working group I to the fourth assessment report of the intergovernmental panel on climate change, In: Solomon, S.; Qin, D.; Manning, M., Chen, Z., Marquis, M., Averyt, K.B., Tignor, M. and Miller, H.L. (Eds.), Intergovernmental Panel on Climate Change: Climate, Cambridge University Press, Cambridge, UK, pp. 3,44.

Wall, E., and Smit, B. (2005), "Climate change adaptation in light of sustainable agriculture", Journal of Sustainable Agriculture, Vol. 27 No. 1, pp. 114. 